\title{
Development and evaluation of prototypes for the ATLAS ITk pixel detector
}

\author{
Florian Hinterkeuser, on behalf of the ATLAS Collaboration ${ }^{a, *}$ \\ ${ }^{1}$ Physikalisches Institut, Universität Bonn, \\ Nußallee 12, 53115 Bonn, Germany \\ E-mail: florian.hinterkeuser@cern.ch
}

\begin{abstract}
The ATLAS inner detector will be replaced by an all-silicon detector for the HL-LHC upgrade around 2025. The innermost five layers of the detector system will be pixel detector layers which will be most challenging in terms of radiation hardness, data rate and readout speed. A serial power scheme will be used for the pixel layers to reduce the material budget and power consumption in cables. New elements are required to operate and monitor a serially powered detector including a detector control system, constant current sources and front-end electronics with shunt regulators. Prototypes for all sections of the ITk pixel detector are built to verify the concept and operate multiple serial power chains as a system test. The evaluation of both the readout of multi-modules and mechanical integration are further aims of the prototyping campaign. In the contribution, results will be presented of this prototyping effort. Moreover, details and features of serial powering for full detector systems will be given.
\end{abstract}

40th International Conference on High Energy physics - ICHEP2020

July 28 - August 6, 2020

Prague, Czech Republic (virtual meeting)

\footnotetext{
${ }^{*}$ Speaker
} 


\section{Introduction}

For the high luminosity upgrade of the LHC, the current tracking detector of the ATLAS [1] experiment will be replaced by completely new all-silicon tracking detector, the ITk, consisting of pixels and strips. For the ITk Pixel detector serial powering [2] has been chosen as a baseline. In this scheme a serial chain of modules is powered by a constant current supply, the supply voltage of the ROCs ${ }^{1}$ is generated by on-chip $\mathrm{SLDO}^{2}$ [3] regulators. As serial powering has not been used in a large scale detector before, it provides major challenges for the electrical and mechanical design of the ITk Pixel detector. A series of prototyping campaigns have been launched to meet these challenges, including large-scale system tests as well as prototyping on chip-level.

\section{ITk Pixel Prototyping}

For each of the 3 subsystems of ITk Pixel, the $\mathrm{IS}^{3}, \mathrm{EC}^{4}$ and $\mathrm{OB}^{5}$, large scale prototypes are in development to cover mechanics, electronics and integration of the final ITk Pixel detector. With modules based on the current generation ROC, the RD53A, not available in sufficient numbers yet, these prototypes are still in early development stages. Prior prototyping, especially the OB demonstrator program and EC Ring-0, with modules based on the FE-I $4 b^{6}$ ROC, already successfully prototyped operation of serial powering chains with lengths of up to 16 modules as well as the operation of several serial powering chains with a common system ground. In addition, valuable input for the system design, integration and further development of the ROC to be used in ITk Pixel was provided. This led to, amongst others, important changes in the specifications for the power supplies for ITk Pixel and the SLDO.

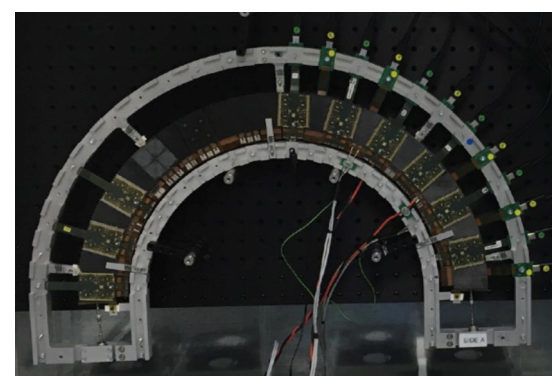

(a) EC Ring-0 loaded with 2 serial powering chains with a total of 12 FE-I4b quad modules on a half ring.

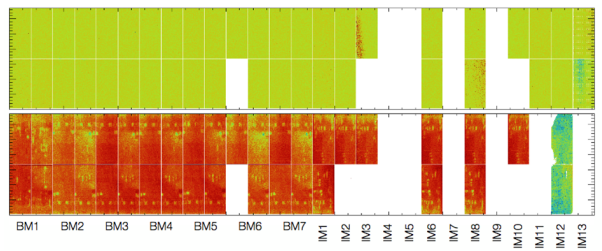

(b) Threshold distribution (top) and source scan (bottom) of the fully loaded A-side of the OB demonstrator with a total of 23 FE-I4b modules. White spaces denote masked ROCs.

\section{Development of the Shunt-LDO voltage regulator}

Previous prototyping campaigns for ITk Pixel and experiences made with the RD53A ROC raised the requirement to add new features to the SLDO. These features include an improved,

\footnotetext{
${ }^{1}$ Readout Chip

${ }^{2}$ Shunt-Low DropOut

${ }^{3}$ Inner System

${ }^{4}$ EndCaps

${ }^{5}$ Outer Barrel

${ }^{6}$ Readout chip used in the ATLAS IBL.
} 
$2 \mathrm{~V}$ tolerant trimmable bandgap reference, an undershunt-current protection to prevent voltage transients in the serial powering chain caused by overloaded SLDOs, an input voltage clamp to prevent damage caused by voltage transients at the SLDO input and a low-power mode to allow module testing during detector integration without available cooling. The updated SLDO design including these new features was prototyped with a series of 3 test chips. All new features have been tested and verified. The radiation hardness of the improved SLDO has been proven during several X-Ray irradiation campaigns with target TIDs $^{7}$ exceeding 1 GRad.

\section{RD53A planar serial powering chain}

Studying of low-level system aspects, such as $\mathrm{HV}^{8}$ distribution schemes or SLDO supply current headroom, require dedicated serial powering prototypes with representative modules based on current generation ROC. One of several existing setups is the RD53A Planar Serial Powering Prototype at Bonn University. This prototype consists of

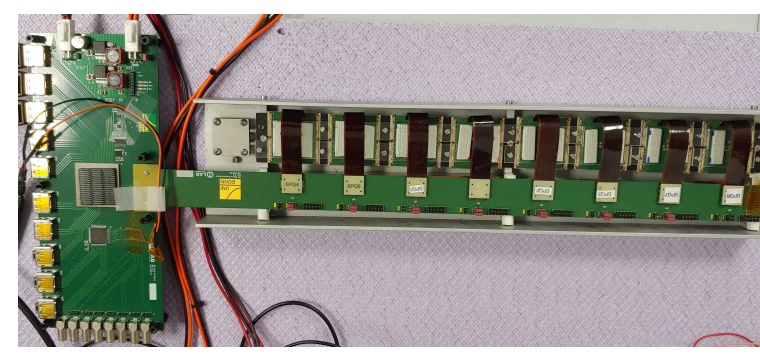

Figure 2: RD53A planar serial powering chain loaded with digital RD53A quad modules on dedicated flex PCBs. up to 8 RD53A quad modules per local support in a serial powering chain powered by a constant current source. Due to limited module availability, the prototype is currently loaded with 8 digital $^{9}$ modules. The services used are dedicated designs for this setup and do not have to comply with constraints for the production services. This allows addition of extra testpoints on the module flex, easing debugging and characterization of the modules in serial powering chain operation. First results in the ongoing commissioning phase are very promising and show a performance of RD53A modules in serial powering chain operation which meets expectations from single-chip testing as well as earlier prototypes with FE-I4b modules.

\section{References}

[1] ATLAS Collaboration, The ATLAS Experiment at the CERN Large Hadron Collider, 2008 JINST 3 S08003

[2] D. Bao Ta et al., Serial powering: Proof of principle demonstration of a scheme for the operation of a large pixel detector at LHC, Nucl. Instrum. Meth. A557 (2006), pp. 445-459, DOI: 10.1016/j.nima.2005.11.115

[3] M. Karagounis et al., An integrated Shunt-LDO regulator for serial powered systems, ESSCIRC'09 Conference, Athens, Proceedings of ESSCIRC '09, 276

\footnotetext{
${ }^{7}$ Total Ionising Dose

${ }^{8}$ High Voltage

${ }^{9}$ Module without a Si-Sensor
} 